\title{
Impact Force when Fabric Inflates at High Speed
}

\author{
Limin BAO and Kiyoshi KEMMOCHI \\ Faculty of Textile Science and Technology, Shinshu University \\ 13-15-1 Tokida, Ueda, Nagano 386-8567, Japan \\ E-mail: baolimin@shinshu-u.ac.jp
}

\begin{abstract}
Occupant protection systems for automobiles are currently highly publicized. An airbag inflating at high speed impacts an occupant with great force. Airbag safety relies on their construction with primarily woven fabrics. We have referred to a typical pressure-time history of an airbag, and developed a device to measure impact load when the fabric airbag inflates at high speed. When interior pressure in the airbag increases, impact load increases. Also, the impact load on a body increases as the distance between the body and airbag decreases. The impact force of an airbag inflating at high speed is simulated by a non-linear FEM combined with an incremental method, where the sample is modeled by a thin elastic shell. An experiment was conducted to verify the theory and this calculation method. Good agreement was obtained between the theoretical and experimental values for two types of fabric samples. This demonstrated that the mathematical technique developed here can satisfactorily predict the impact force when an airbag inflates at high speed and that the impact force depends appreciably on the mechanical properties of fabrics.
\end{abstract}

Keyword: Impact load, Fabrics, Airbag, Evaluation, Inflation, Polymeric materials

\section{Introduction}

Occupant protection systems for automobiles are currently highly publicized. The death rate decreases in collisions involving cars equipped with airbag systems. However, when a fabric airbag inflates at high speed, it applies a large impact force to occupants. The percentage of injuries, such as bruises and scratches, caused by the contact between occupants and airbags is very high. ${ }^{1)}$ Therefore, to decrease the impact force, the mechanism of incidence of impact force needs investigation. Development of airbags that are non-injurious to humans is essential.

In this field, R. Keshavaraj ${ }^{2)}$ suggested a device for measuring the quasi-dynamic permeability of fabrics used in airbags. Wang and $\mathrm{Bao}^{3)}$ used the reflected shock wave theory to measure the permeability of airbag fabrics in a shock wave under the same conditions in which an airbag system inflates. In a shock wave, the airbag's permeability is lower than its static and quasi-dynamic permeability, proving the possibility of a non-coated airbag.

Simamura ${ }^{1)}$ reported that occupants received some abrasion on their faces and heads after contacting an opened airbag in a collision. $\mathrm{We}^{4)}$ presume that the frictional force between the airbag and occupant causes the abrasion; therefore, we investigated frictional characteristics of the airbag's materials under the impact load.

There are many studies on the impact forces of fabric airbags. Kobayashi ${ }^{5)}$ suggested an air bag and human collision model, and simulations were conducted to observe the impact characteristics of airbags. However, our experimental results ${ }^{4)}$ differed from his.

Manufacturers ${ }^{6-8)}$ use original airbags and dummies to 
study the airbag folding process and inflation time, and to develop optimum airbag systems. However, there are no studies concerning the effect of inflation pressure and physical characteristics on the impact against bodies when airbags inflate at high speed. Therefore, there are many unknowns in this field.

In this research, we developed a device to measure the impact force when the fabric airbag inflates at high speed to provide basic data for a more advanced airbag design. We used this device to measure the impact force in different conditions and fabrics. To predict impact load upon a body, a nonlinear FEM considering the large lateral deformation and contact was performed to solve them. We conducted simulations to observe the effect of various parameters on impact force when an airbag inflates at high speed.

\section{Theoretical investigation}

\subsection{Basic equations}

Fabric is complicated structure to consist of the yarn which is fiber or the aggregate. Though fabric isn't a continuous body, we took the average characteristic comprehensively and treated it on the basis of membrane theory to simplify analysis. This model is suggested with Hearle $^{9)}$, and it is used now by a lot of researchers ${ }^{10)}$. The model of deformed fabric is shown in Fig. 1, where $p$ is the distributed load. For large deformation, the relationship between strain $\left(\varepsilon_{x}, \varepsilon_{y}, \varepsilon_{x y}\right)$ and displacement $(u, v, w)$ in $\mathrm{x}, \mathrm{y}$, and $\mathrm{z}$ directions of a local coordinate system are shown:

$$
\left\{\begin{array}{c}
\varepsilon_{x} \\
\varepsilon_{y} \\
\varepsilon_{x y}
\end{array}\right\}=\left\{\begin{array}{c}
\frac{\partial u}{\partial x} \\
\frac{\partial v}{\partial y} \\
\frac{\partial u}{\partial y}+\frac{\partial v}{\partial x}
\end{array}\right\}+\left\{\begin{array}{c}
\frac{1}{2}\left(\left(\frac{\partial u}{\partial x}\right)^{2}+\left(\frac{\partial v}{\partial x}\right)^{2}+\left(\frac{\partial w}{\partial x}\right)^{2}\right) \\
\frac{1}{2}\left(\left(\frac{\partial u}{\partial y}\right)^{2}+\left(\frac{\partial v}{\partial y}\right)^{2}+\left(\frac{\partial w}{\partial y}\right)^{2}\right) \\
\frac{\partial u}{\partial x} \frac{\partial u}{\partial y}+\frac{\partial v}{\partial x} \frac{\partial v}{\partial y}+\frac{\partial w}{\partial x} \frac{\partial w}{\partial y}
\end{array}\right\}
$$

The constitutive equation is given by

$$
\left\{\begin{array}{c}
\sigma_{x} \\
\sigma_{y} \\
\sigma_{x y}
\end{array}\right\}=\left[\begin{array}{lll}
Q_{11} & Q_{12} & Q_{13} \\
Q_{12} & Q_{22} & Q_{23} \\
Q_{13} & Q_{23} & Q_{33}
\end{array}\right]\left\{\begin{array}{c}
\varepsilon_{x} \\
\varepsilon_{y} \\
\varepsilon_{x y}
\end{array}\right\}
$$

$$
\{\sigma\}=[D]\{\varepsilon\}
$$

where $Q_{11} \quad \cdots \cdots \quad Q_{33}$ are the in-plain stiffnesses, $\sigma_{x}$ and $\sigma_{y}$ are the stresses in $x, y$ directions, respectivery, and $\sigma_{x y}$ is the shearing stress.

The stiffnesses are given by

$Q_{11}=\frac{E_{L}}{1-v_{L T} v_{T L}} \times l^{4}+2\left(\frac{v_{T L} E_{L}}{1-v_{L T} v_{T L}}+G_{L T}\right) \times l^{2} m^{2}+\frac{E_{T}}{1-v_{L T} v_{T L}} \times m^{4}$ $Q_{66}=\left(\frac{E_{L}}{1-v_{L T} v_{T L}}+\frac{E_{T}}{1-v_{L T} v_{T L}}-2 \frac{v_{T L} E_{L}}{1-v_{L T} v_{T L}}-2 G_{L T}\right) \times l^{2} m^{2}+G_{L T}\left(l^{4}+m^{4}\right)$ $, l=\cos \theta, \quad m=\sin \theta$

where $E_{L}$ and $E_{T}$ are the tensile moduli in warp and weft directions, respectivery, $G_{L T}$ is the apparent shearing moduli, $v_{L T}, v_{T L}$ are apparent Poisson's ratio, and $\theta$ is the angle between warp and $x$ axis.
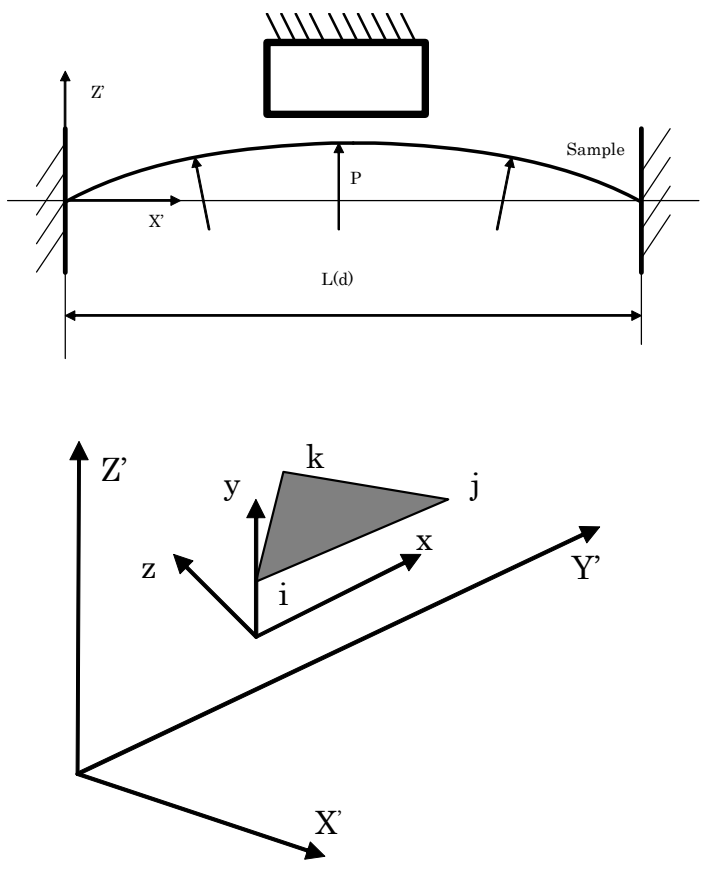

Fig.1 Geometry of fabrics element.

\subsection{Analysis methods}

An incremental method ${ }^{11)}$ was employed in an FEM calculation of lateral deformation of fabrics. We applied critical-flow theory using a convergent nozzle to calculate the increase in mass flow rate, since the difference in air pressure between the tank and the airbag were considered. Using this mass flow rate, pressure increase in an airbag in an adiabatic state was calculated, and airbag shape was 
derived using the FEM method. The pressure increase in the airbag with this increased volume is then recalculated, and this calculation is repeated to convergence.

The airbag contacts a load sensor disk when it inflates and the impact forces are generated. In calculating this impact force, we did not include the inertia force of the airbag fabrics because the fabrics were thin and the mass was small. Between increases in FEM, we used total tension $T$ of fabric and total air pressure to calculate contact forces with the load sensor disk, as shown in Fig. 2.

The sample is divided again before an increase so that the sample node aligns with (A) at the end of the disk because the fabric deformation is large.

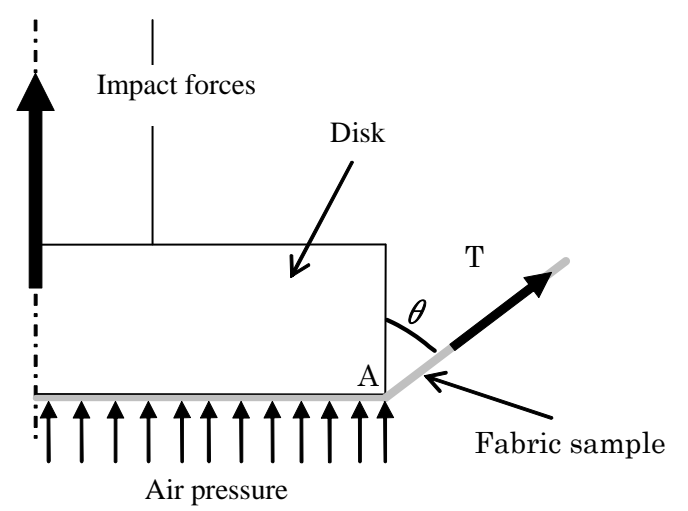

Fig.2 Impact model for the simulation.

\section{Experiment}

\subsection{Experimental device and method}

Figure 3 depicts a schematic diagram of the experiment's equipment. The air was adjusted to a fixed pressure by an air compressor and regulator, then stored in an air tank. The airbag fabric was tightly set on the head and inflated by the compressed air, which was controlled by a high-cycle electromagnetic valve (Koganei Co. Ltd., HV160E1-2). The dynamic pressure was measured by a pressure sensor (Kyowa Co. Ltd., PGM-10KC). The inflating fabric collides with the disk, and impact load is measured with a load sensor (Kyowa Co. Ltd., LU-100KSB34D). Impact load of a load cell disk and interior pressure are recorded on a personal computer through two signal conditioners (Kyowa Co.,
Ltd., CDV-700A) and a high-speed A/D board. When the fabric inflates at high speed, displacement of the fabric's center is measured by a laser sensor (Keyence Co. Ltd., LB1000) on this device, and the interior pressure is measured simultaneously.

Referring to the results of pressure from $\mathrm{R}$. Keshavaraj $^{2)}$ a fabric sample was fixed with eight bolts (M8) to withstand the pressure. We installed a double $\mathrm{O}$ ring on the sample part that was fixed. Considering the overall ratio of the size of occupants' faces and airbags, and the characteristics of the high-cycle electromagnetic valve, the fabric sample diameter was set to $110 \mathrm{~mm}$ and the load cell disk diameter to $30 \mathrm{~mm}$.

The setting pressure and the distance between the load cell disk and samples were changed, and the experiment was repeated three times under the same conditions.

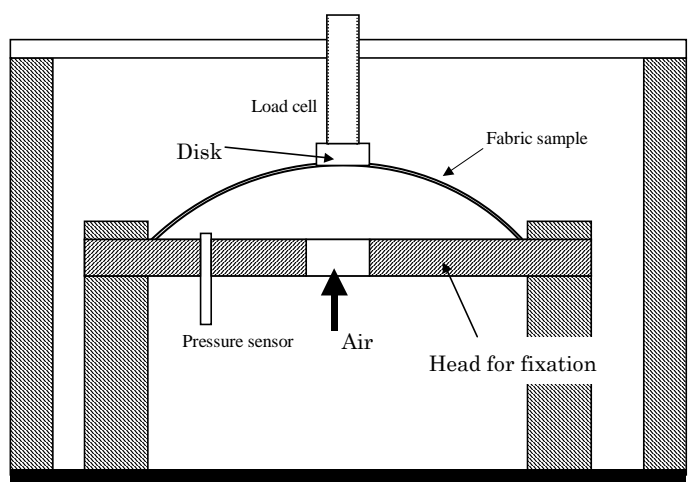

Fig. 3 Experiment apparatus for measuring impact forces when an air bag is inflated at high speed.

\subsection{Samples}

All the samples shown in Table 1 were non-coated fabric used in automobile airbags. The standard sample was provided by Toyota Boshoku Co., Ltd.; the sample material was Nylon 66.

Table 1 Physical characteristics of fabric samples

\begin{tabular}{lcccc}
\hline Type No. & Material & $\begin{array}{l}\text { Dtex } \\
\text { /Filaments }\end{array}$ & $\begin{array}{l}\text { Ends and } \\
\text { Picks/inch }\end{array}$ & Weave \\
\hline N4256 & Nylon 66 & $470 / 72$ & $55 \times 55$ & Plain \\
\hline N3562 & Nylon 66 & $350 / 73$ & $63.5 \times 60.5$ & Plain \\
\hline
\end{tabular}

Tension tester (Shimazu AG20KND) was used to measure tensile stiffness of the samples. The width and the span of the samples were each $50 \mathrm{~mm}$ and $200 \mathrm{~mm}$. 
We tested each at the strain rate of $1 \% /$ minute, for warp and weft direction. The Poisson's ratio and shear stiffness of fabric in calculations were obtained by the paper ${ }^{12-13)}$. Table 2 presents the measurement results of fabrics.

Table 2 Mechanical properties of fabric samples

\begin{tabular}{ccccc}
\hline $\begin{array}{c}\text { Type } \\
\text { No. }\end{array}$ & $\begin{array}{c}\mathrm{E}_{\mathrm{L}} \\
(\mathrm{N} / \mathrm{m})\end{array}$ & $\begin{array}{c}\mathrm{E}_{\mathrm{T}} \\
(\mathrm{N} / \mathrm{m})\end{array}$ & $\begin{array}{c}\mathrm{G} \\
(\mathrm{N} / \mathrm{m})\end{array}$ & $v_{L T}$ \\
\hline N4256 & 103.86 & 48.95 & 2.10 & 0.682 \\
\hline $\mathrm{N} 3562$ & 82.34 & 58.57 & 1.99 & 0.795 \\
\hline
\end{tabular}

\section{Results and Discussion}

\subsection{Impact force at high speed}

The fabric sample was fixed and inflated by the compressed air. The impact force was measured by the device mentioned in section 3.1.

Figure 4 presents the results of an experiment investigating the relationship between time and interior pressure of an airbag and displacement of a sample center when the air tank pressure was 0.3MPa. The test sample is N4256. The results of two experiments are roughly the same, with a few dispersions of data. As illustrated in Fig. 4 , the time of the result is the same level as the inflating time of original airbag systems ${ }^{2}$. We averaged displacement data from 300 to 400msec in Fig. 4 and defined it as the maximum displacement of the sample center. We also averaged data of interior pressure from 300 to 400 msec in Fig. 4 and defined it as the maximum interior pressure.

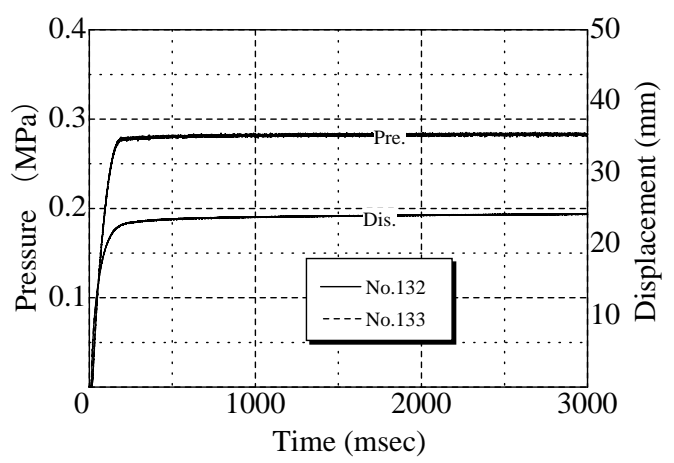

Fig. 4 Time dependence of interior pressure and displacement, and impact load when an airbag is inflated.

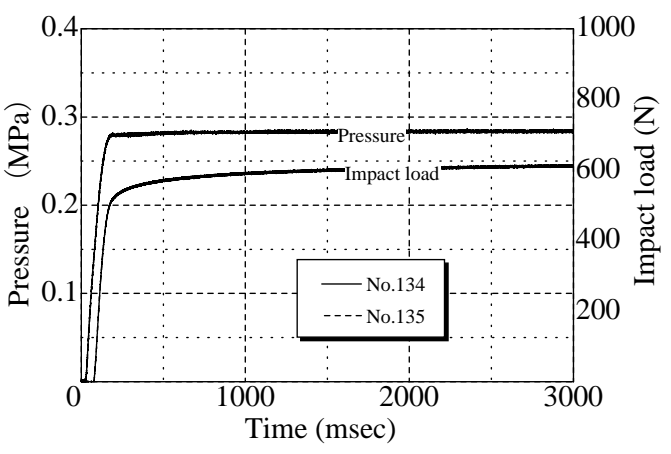

Fig. 5 Time dependence of pressure and impact load when an air bag is inflated at high speed. (Sample: N4256, $\mathrm{h}=15.8 \mathrm{~mm}$ )

Figure 5 illustrates the time dependence of impact force versus the load cell disk and interior pressure in a fabric, when the diameter of the load cell disk is $30 \mathrm{~mm}$ and the vertical distance (h) with load cell disk and fixed head is $15.8 \mathrm{~mm}$. The right axis measures impact load, and the left axis is interior pressure in a sample. The load cell disk collides after the sample is inflated. The results of two experiments are roughly the same, with a few dispersions of data. Figure 5 indicates that the force on a load sensor will gradually increase. We averaged data of impact loads with 300 to $400 \mathrm{~m}$ sec in Fig. 5 and defined it as the maximum impact load (Impact load $\operatorname{lmax}_{\max }$.

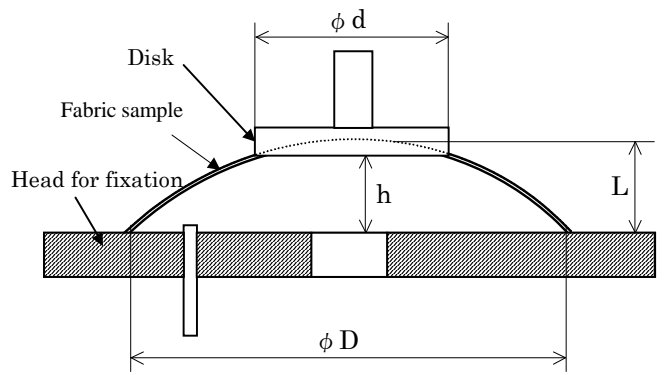

Fig. 6 Contact point of fabric with disk.

\subsection{Indenting ratio of load cell disk (i)}

The interior pressure and the vertical distance $(h)$ between the load cell disk and the head have an effect on the maximum impact load. As shown in Fig. 6, the indenting ratio of load cell disk (i) is defined as follows.

$$
i=(L-h) / L
$$

Here, $L$ is the maximum displacement at the center of the sample when the load cell disk does not contact it and is 
calculated from Fig. 4.

Pressure ratio ( $\mathrm{P}_{\text {load }} /$ Pre.) is defined as follows.

$$
\mathrm{P}_{\text {Load }} / \text { Pre }=\text { Impact load } \max _{\text {max }} /\left(A^{*} \text { Pressure }\right)
$$

Here, $A$ is the area of a load sensor disk, and the pressure is the atmospheric pressure in the sample. We calculate the maximum impact load by expression (6) and summarize it in Fig. 7 and Fig.8. Figure 7, 8 illustrates the relationship between the indenting ratio (i) and pressure ratio $\left(\mathrm{P}_{\text {load }} /\right.$ Pre.). The dots represent experiment values. The relationship roughly describes a single curve, independent of vertical distance $(h)$ and interior pressure.

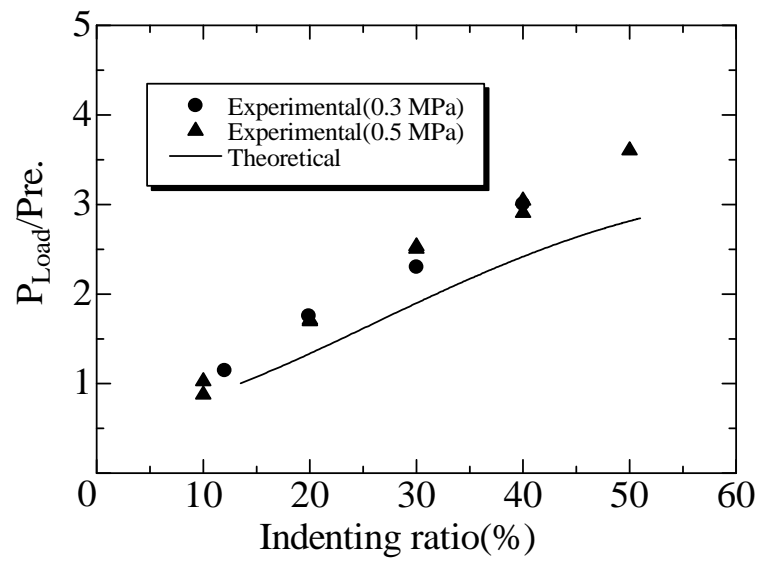

Fig. 7 Relationship between indenting ratio (i) and pressure ratio ( $\mathrm{P}_{\text {load }} /$ Pre.); (N4256)

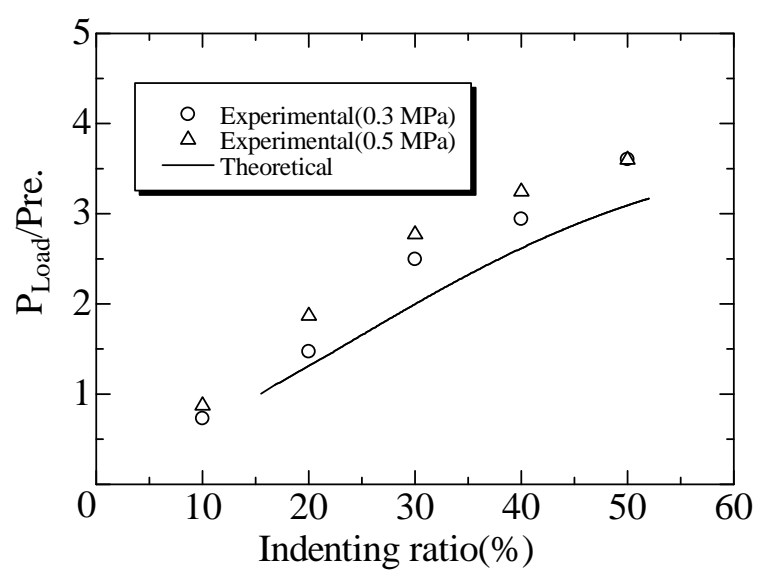

Fig. 8 Relationship between indenting ratio (i) and pressure ratio ( $\mathrm{P}_{\text {load }} /$ Pre.), (N3562)

\subsection{Comparison with the experimental result}

Figure 9 uses this study's calculation method and depicts an example of its shape. Because the sample was symmetric, a triangular element was used for sample 1/4, and it was divided into 780 elements. The contact part of the sample was more finely divided. We used the mechanical properties of N4256 in Table 2 for the calculations.
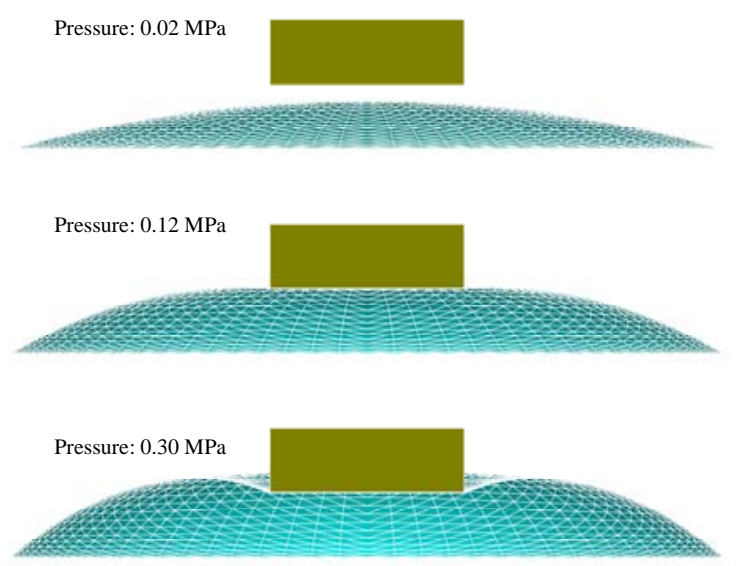

Fig.9 Configuration of the airbag and disk obtained by calculation.

Figures 7 and 8 summarize the calculated results using the data from Table 2 and our calculation method. The graph plots the relationship between the indenting ratio (i) and the pressure ratio $\left(\mathrm{P}_{\text {load }} /\right.$ Pre.). The line represents theoretical values. The samples represented in Figs. 7 and 8 are N4256 and N3562. There is agreement between the experimental and theoretical values. The tendency in variations of the two materials also agrees, confirming the applicability of the formula suggested in this research.

\subsection{Effect of mechanical properties on impact force}

Using the calculation method suggested in this research, we conducted simulations to observe how the parameters affect the impact forces when fabrics inflate at high speed. Figures 10, 11 and 12 are the results of simulations.

Figure 10 displays the relationship between the maximum impact load and the air pressure. We used the mechanical properties of sample N4256 in Table 2. The mean $(E)$ of the tensile moduli of the fabrics N4256 in warp and weft directions was adopted. When air pressure 
in an airbag increases, the maximum impact load applied to the load sensor disk increases. In Fig. 10, we increased h (vertical distance between load cell disk and fixed head) by $5,10,15,20$ and $25 \mathrm{~mm}$ while keeping the other parameters $\left(E, G_{L T}, v_{L T}\right)$ constant, illustrating that the maximum impact load decreased as $\mathrm{h}$ increased. It is clear that distance between an airbag and the occupant is important.

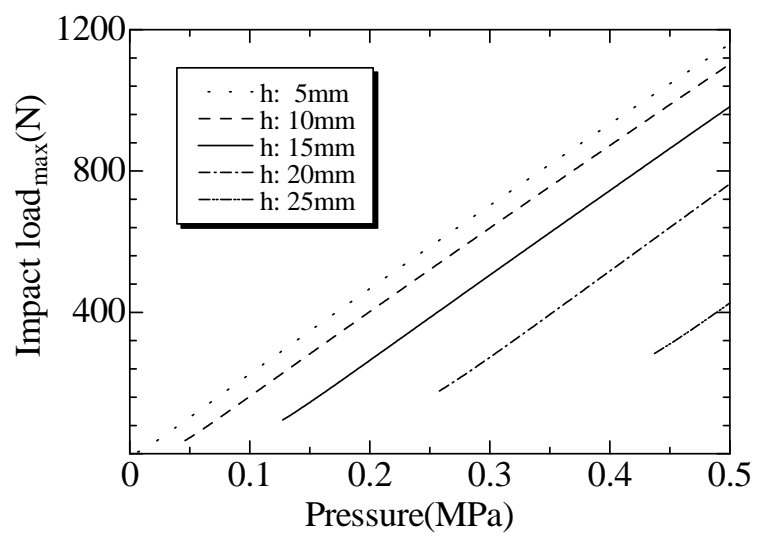

Fig. 10 Relationship between air pressure and maximum impact load in variation of h.

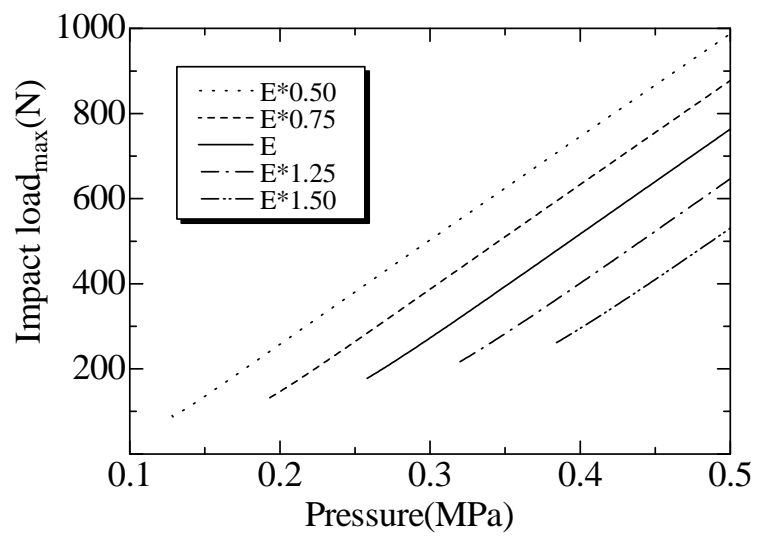

Fig. 11 Relationship between air pressure and maximum impact load in variation of $\mathrm{E}$.

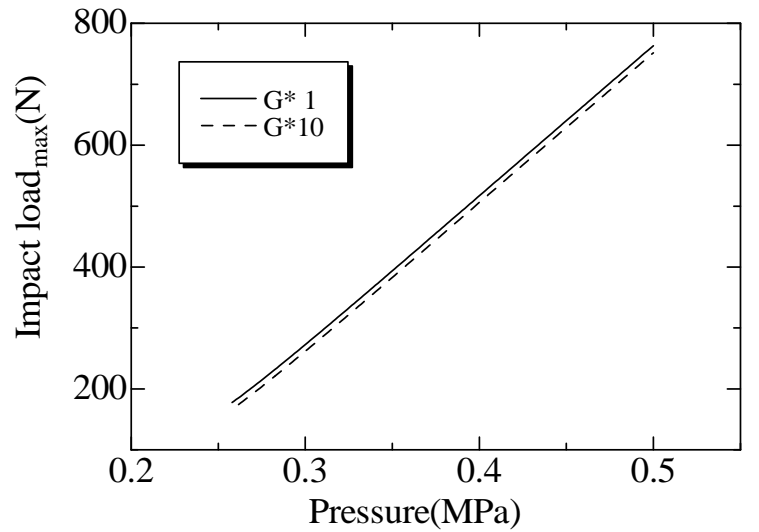

Fig. 12 Relationship between air pressure and maximum impact load in variation of G.

In Fig. 11, we increased $E$ by 1.25 and 1.50 times then reduced it by 0.75 and 0.50 while keeping the other parameters ( $h, G_{L T}, v_{L T}$ ) constant. The vertical distance (h) between the load cell disk and fixed head is $20 \mathrm{~mm}$. We observed that the maximum impact load decreased as $E$ increased. As Fig. 9 indicates, with similar air pressure in an airbag, displacement and indenting ratio increase as stiffness of fabric decrease, and the maximum impact load increases.

The apparent shearing moduli of fabrics are considerably changed by rubber or vinyl coatings. In Fig. 12, we increased $G_{L T}$ by 10 times while keeping the other parameters ( $\left.h, E, v_{L T}\right)$ constant. The results indicated that the maximum impact load decreased as $G_{L T}$ increased. However, it has less effect on the maximum impact load than $E$. $v_{L T}$ has the same tendency. This is because the research model is symmetric ${ }^{10)}$.

\section{Conclusions}

We studied the impact force when a fabric airbag inflates at high speed to provide basic data for a more advanced airbag design. Impact force when an airbag inflates at high speed is simulated by a non-linear FEM combined with an incremental method.

We developed an experimental device to measure the impact force when a fabric airbag inflates at high speed to 
verify the theory and this calculation method. Good agreement was obtained between the theoretical and experimental values of two types of fabric samples. This demonstrates that the mathematical technique developed here can satisfactorily predict the impact force when an airbag inflates at high speed and that the impact force depends appreciably on the mechanical properties of fabrics.

\section{Acknowledgement}

This work was supported by a Grant-in-Aid for COE Research (10CE2003) and Scientific Research (C)(2) 13650081 by the Ministry of Education, Culture, Sports, Science and Technology of Japan.

\section{Literature Cited}

1. M. Simamur, H. Sarawatari, S. Yabushita, Society of automotive engineers of Japan annual meeting preprints, 20005270(2000).

2. R.Keshavaraj, R.W. Tock and D. Haycook, Analysis of fabrics used in passive restraint systems - airbags, J. Text. Inst. 87 Part 1, No.3, 554-571(1996).

3. X. Wang, L. Bao, M. Nakazawa, T. Kawamura and T. Kobayashi, Determine Permeability of Airbag fabrics with Shock Tube Experiment, J. Text. Inst. (in press).

4. L. Bao, R. Takamasu, M. Sakurayi, K. Kemmoch and M. Nakazawa, Frictional Characteristic of SRS Air Bag Materials under the Impact Load, The $2^{\text {nd }}$ International Conference on Advanced Fiber/textile Materials, JAPAN, P162(2002).

5. K.Kobayashi, M.Morisawa, A Study of Optimal Design for Structural Elements of Airbag-Seatbelt System, JSAE Transaction, Vol.29,No1,135-140 (1998)

6. K. Seo, Holding Film-Technology of air bag system for automobile. Tran. JP.N Soc. Mech. Eng, Vol.102, No.926, 35-38 (1999).

7. T.Sugasawa, Application of the synthetic polymer materials for the air bag system. Function Materials, 17-3, 38-44(1997).

8. Striking, Adouble blow for safety - A revolutionary airbag system due to be revealed next year on the Renault Laguna. Automotive Engineer, London, Vol.24, No.7, 39-41(1999).

9. W.J.Shanahan and W.S.Hearle, Characterizing the Elastic Behavior of Textile fabrics in Complex Deformations, Text. Res .J., 69,495- 505(1978).

10. L. Bao, M. Takatera and A. Shinohara, Analysis of Large Non-Linear Elastic Deformation of Fabrics, $J$. Text. Inst.,93, part 1, No.4 410-419(2002).

11. O.C.Zienkiewicz and R.L.Taylor, The Finite Element Method, $4^{\text {th }}$ Ed.,McGraw- hill(1991)

12. L. Bao, M. Takatera and A. Shinohara, Determining the Apparent Shear Rigidity of Textile fabrics by Uniaxial Tensile Test, Sen’i Gakkaishi, 53, 38-44 (1997).

13. L. Bao, M. Takatera and A. Shinohara, Error Evaluation on Measuring the Apparent Poisson's Ration of Textile Fabrics by Uniaxial Tensile Test Sen'i Gakkaishi, 53, 20-26 (1997) 